\title{
Essais sur modèle de rideaux de soutènement; confrontation à diverses méthodes de calcul
}

\author{
Experiments on retaining wall models; \\ confrontation with different calculation methods
}

F. MASROURI
Maître de conférences, Laboratoire de Géomécanique, ENSG Nancy*

R. KASTNER

Professeur, Laboratoire de Géotechnique, INSA de Lyon**

Rev. Franç. Géotech. n 55 , pp. 17-33 (avril 1991)

\section{Résumé}

On présente des essais sur modèle bidimensionnel de rideaux de soutènement en considérant l'influence des conditions de butonnage ou d'ancrage (nombre, raideur, précontrainte). Le comportement de ces rideaux est analysé et comparé aux résultats des méthodes de calcul traditionnelles en plasticité ainsi qu'à ceux obtenus par le calcul suivant l'hypothèse du module de réaction.

\section{Abstract}

We present experiments on a bidimensionnal model of flexible retaining walls and analyse the influence of anchors or struts (number, rigidity, prestressing). The behaviour of these walls is analysed and compared to the results obtained through conventional plasticity calculation methods and also to those obtained from the reaction module hypothesis. 


\section{PRÉSENTATION}

La multiplication des travaux d'aménagement en site urbain a entraîné, au cours des deux dernières décennies, un fort développement des travaux de soutènement utilisant la technique de la paroi moulée ou divers procédés dérivés. Dans un contexte urbanisé, afin de préserver le bâti existant, on impose généralement une limitation stricte au déplacement de ces ouvrages par mise en ceuvre de niveaux multiples de butons ou d'ancrages souvent précontraints.

Les méthodes de calcul traditionnelles en plasticité développées pour des ouvrages très flexibles où d'importantes déformations étaient tolérées ne sont plus adaptées à ces ouvrages au déplacement limité. Dans ces conditions, de nouvelles méthodes de calcul ont été élaborées visant à prendre en compte, parfois empiriquement, la relation pression des terresdéplacement. Ainsi, ROWE (1952) a proposé de corriger la méthode du rideau ancré simplement buté en considérant les influences respectives de la flexibilité du rideau et de la raideur du sol.

A la fin des années 60 , le développement des moyens de calcul informatique a permis à plusieurs auteurs, en particulier HALLIBURTON et al. (1968), BOUDIER et al. (1970), FAGES et al. (1971) de proposer des logiciels de calcul des rideaux flexibles reposant sur l'hypothèse de WINKLER (1867) adaptée au cas particulier des soutènements. Par la suite, on a également utilisé les logiciels généraux utilisant la méthode de éléments finis (MONNET et al., 1985) voire développé des logiciels MEF spécifiques aux soutènements flexibles (BALAY et al., 1985).

Actuellement les logiciels de calcul des soutènements flexibles reposant sur l'hypothèse de WINKLER sont très largement utilisés en France sans que soient toujours bien cernées leurs limites liées à lhypothèse très simplificatrice du module de réaction. Des éléments de réponse ont pu être apportés par diverses expérimentations en vraie grandeur (GIGAN, 1979 ; KASTNER, 1982).

Toutefois de telles expérimentations constituent des tests de validation insuffisants dans la mesure où de trop nombreux paramètres y sont mal cernés. De même, les résultats expérimentaux sur modèles réduits existants, notamment ceux de ROWE (1952-55) ne sont pas représentatifs des ouvrages actuellement utilisés en site urbain.

C'est dans ce contexte que nous avons réalisé une campagne d'essais sur modèle de soutènement flexible maintenu par des butons ou ancrages de raideur et précontrainte variables (MASROURI, 1986) dont les résultats servent de base au test de la méthode de calcul basé sur l'hypothèse du module de réaction, afin d'en mettre en évidence les limites.

\section{LE MODÈLE}

Afin de permettre une répétition aisée des essais, cette campagne expérimentale a été réalisée sur un modèle utilisant le matériau analogique bidimensionnel de SCHNEEBELI (fig. 1). Plus facile à mettre en ceuvre que les modèles en sable, il supprime les effets de bord dus aux parois vitrées et permet l'accès au champ de déplacement par la technique stéréophotogrammétrique (BACOT et al., 1984).

\subsection{Le matériau analogique}

Décrit notamment par SCHNEEBELI (1957) ce matériau pulvérulent bidimensionnel est obtenu par l'empilement de petits rouleaux. Dans le modèle utilisé, les rouleaux de $60 \mathrm{~mm}$ de long et de 3,4 et $5 \mathrm{~mm}$ de diamètre sont en acier inox. Les propriétés mécaniques à la rupture de ce « sol » bidimensionnel mesurées lors d'essais biaxiaux (analogues aux essais triaxiaux de révolution) sont les suivants :

angle de frottement interne : $\phi=21^{\circ}$

cohésion : $\mathrm{c}=0$

La densité élevée de ce matériau $\left(\gamma_{d} / \gamma_{w}=6,5\right)$ est facilement reproductible.

\subsection{Le rideau flexible}

Il est constitué d'une plaque de Dural de $800 \mathrm{~mm}$ de hauteur et $12 \mathrm{~mm}$ d'épaisseur, équipée de 30 jauges de déformation permettant de déterminer avec précision la courbure du rideau sur 20 niveaux. La flexibilité de cette paroi suivant la définition de ROWE

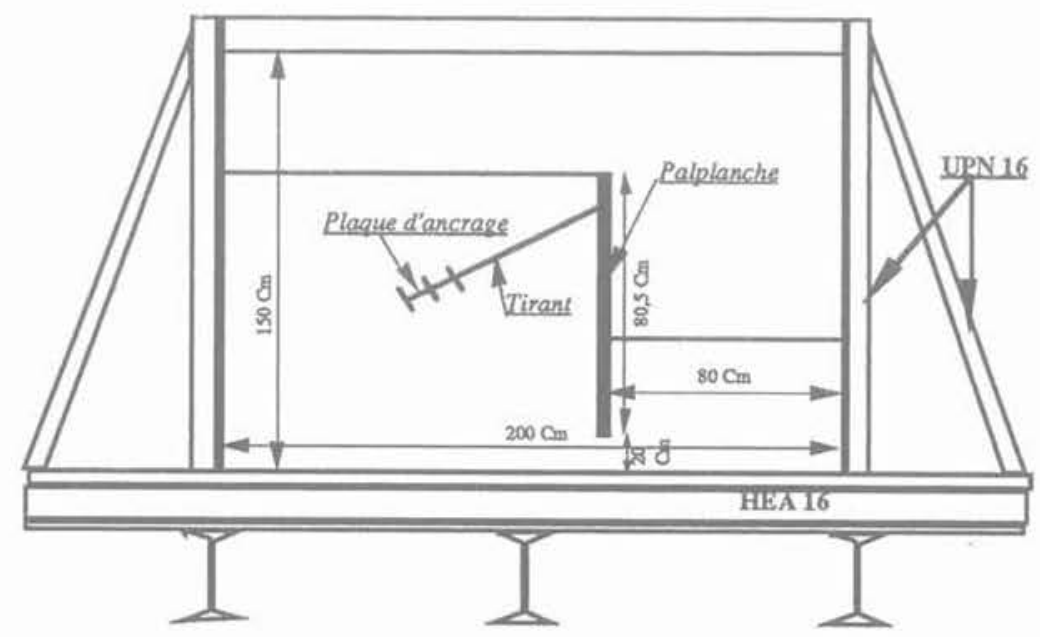

Fig. 1. - Schéma du modèle.

Fig. 1. - Diagram of model. 
(1972) la situe dans le domaine des rideaux peu flexibles tels que ceux utilisés pour les soutènements en site urbanisé (fig. 2).

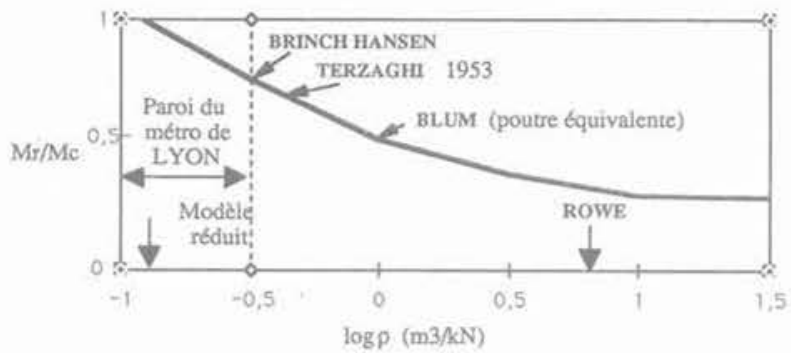

Fig. 2. - Situation du modèle dans l'échelle de flexibilité de ROWE (1972).

Fig. 2. - Position of the model

in the ROWE flexibility scale (1972)

\subsection{Butons-Ancrages}

Lors de l'excavation, le soutènement est maintenu par un ou deux niveaux de butons (photos 1 et 2) ou d'ancrages dont on a fait varier la raideur et la précontrainte. Pour simuler un bulbe d'ancrage injecté, un corps d'ancrage constitué de profils en $\mathrm{H}$ est noyé dans le massif et relié à la tête du rideau par deux tirants situés de part et d'autre du modèle.

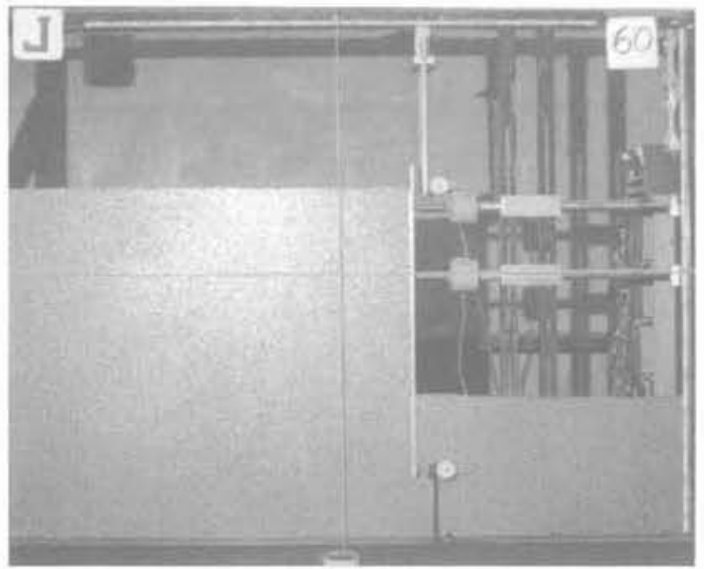

Photo 1 - Ecran avec 2 butons.

Photo 1 - Sheet pile with 2 struts.

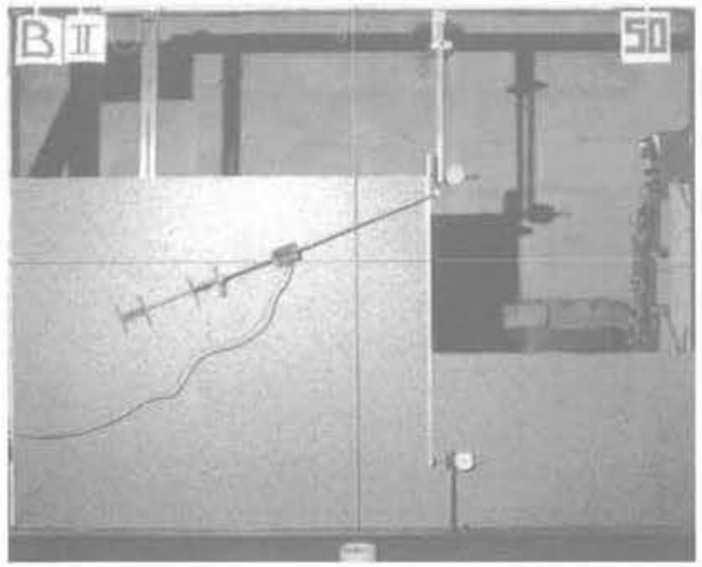

Photo 2 - Ecran avec 1 ancrage. Photo 2 - Anchored sheet pile.

\section{EXPLOITATION DES MESURES}

\subsection{Mesures effectuées}

A chaque étape d'excavation, on effectue l'ensemble de mesures suivantes:

- déplacement en tête et en pied du rideau mesuré à l'aide de comparateurs au $1 / 100^{e} \mathrm{~mm}$;

- effort dans les ancrages ou les butons par l'intermédiaire de capteurs dynamométriques à jauges :

- mesure de la courbure à partir des 30 jauges extensométriques collées sur l'intrado et l'extrado du rideau ;

- prise de clichés à laide d'un appareil Hasselblad $6.6 \mathrm{~cm}$ pour exploitation éventuelle par stéréophotogrammétrie.

\subsection{Détermination des diagrammes de déformée, moment fléchissant et pression différentielle}

Ces trois diagrammes peuvent être déduits des mesures de courbure, directement pour le moment fléchissant, après double intégration et double dérivation pour la déformée et la pression différentielle. Toutefois, si l'intégration numérique est peu sensible aux incertitudes expérimentales attachées au diagramme d'origine, il n'en est pas de même pour la dérivation qui a tendance à amplifier les erreurs. Aussi un logiciel spécifique (BOISSIER et al., 1978) a-t-il été développé pour ces opérations : les résultats expérimentaux sont lissés par la méthode de moindres carrés à l'aide de polynômes permettant la prise en compte des points singuliers des divers diagrammes (niveau de butonnage, fond de fouille). Les opérations de double intégration et double dérivation sont ensuite effectuées à partir de ce diagramme lissé. Des tests réalisés sur divers exemples théoriques ont confirmé la pertinence de cette démarche.

\subsection{Mesure du champ de déplacement par photogrammétrie}

A chaque phase de l'expérimentation (excavation, pose de buton...), un cliché du modèle est réalisé. L'examen simultané de deux clichés au stéréorestituteur fait apparaitre un relief apparent proportionnel en chaque point au déplacement entre les deux phases analysées. On peut ainsi reconstituer le champ de déplacement du sol sur l'ensemble du modèle. En pratique, seuls quelques essais ont été ainsi analysés en raison du cout de l'opération.

\subsection{Reproductibilité des essais}

Elle a été testée par comparaison de deux essais identiques effectués par deux expérimentateurs différents. La figure 3 présente à titre d'exemple les résultats obtenus pour $50 \mathrm{~cm}$ d'excavation. On note l'excellente reproductibilité, tant pour le moment fléchissant déduit directement des mesures de courbure, que pour la pression différentielle obtenue après lissage et double dérivation. 
De même les différences d'effort dans les butons restent inférieures au pourcent si l'on excepte la phase de mise en place et diminuent avec la progression de l'excavation.

\section{RÉSULTATS EXPÉRIMENTAUX}

\subsection{Programme d'essai}

Le programme d'essai a été conçu afin que ses résultats constituent une base complète pour les tests de validation des méthodes de calcul. Dans cette optique les configurations étudiées ont été choisies afin que le sol soit sollicité de manière suffisamment variée pour que les limites des méthodes de calcul puissent être mises en évidence. Ainsi une quarantaine d'expérimentations ont été réalisées sur le même rideau, en faisant varier les conditions d'étaiement :

- rideau non ancré ;

- rideau butonné avec:

- 1 ou 2 butons passifs de diverses raideurs;

- 1 ou 2 butons précontraints de diverses raideurs ;

- rideau ancré avec ancrage de diverses longueurs et inclinaisons, précontraint ou non.

Les tableaux présentant les caractéristiques de ces essais sont donnés en annexe.

On a ainsi testé des comportements variés allant de la rotation du rideau par rapport à sa base à la rotation par rapport au sommet, en passant par les ruptures par insuffisance d'ancrage et les rideaux maintenus par deux niveaux de butons précontraints.

\subsection{Rideau non ancré}

La figure 4 présente l'évolution des déformées et du déplacement en tête avec la progression de l'excavation. On note que les déplacements en tête restent faibles jusqu'à $29 \mathrm{~cm}$ d'excavation (ce qui correspond au maintien de l'encastrement en pied) pour s'accélérer ensuite avec un seuil de rupture pour $f=31,5 \mathrm{~cm}$.
Le calcul suivant le schéma classique du rideau encastré non ancré (avec $\delta / \phi=-2 / 3$ en butée) conduit à une excavation à la rupture de $37,5 \mathrm{~cm}$ et une fouille de $27 \mathrm{~cm}$ pour l'ouvrage en "service "calculé avec un coefficient de sécurité de 2 sur la butée. Ce schéma de calcul qui admet un passage brutal de la poussée à la contre-butée à l'arrière du rideau s'avère ainsi trop optimiste.

Le schéma simplifié où la contre-butée est remplacée par une force concentrée au point de * rotation * du rideau reste lui aussi trop optimiste (h rupture $=36 \mathrm{~cm}$ ).

\subsection{Rideau maintenu par un niveau de butons}

Après une première excavation de $10 \mathrm{~cm}$, le buton est placé à $4 \mathrm{~cm}$ du sommet, puis précontraint. L'excavation est ensuite poursuivie par pas de 10 puis $5 \mathrm{~cm}$ jusqu'à la rupture. Une douzaine d'essais ont été réalisés en faisant varier la raideur et la précontrainte du buton.

\subsubsection{Rideau avec 1 buton très déformable non précontraint (fig. 5)}

Avant de commenter les résultats généraux de ces essais, trois exemples significatifs sont présentés. Suivant la théorie du rideau ancré simplement buté (avec $\delta / \phi=-1$ ), la rupture survient pour une excavation h de $55,5 \mathrm{~cm}$ alors qu'elle serait de $44,6 \mathrm{~cm}$ pour l'ouvrage en service (avec un coefficient de sécurité de 2 sur la butée).

Notons tout d'abord que sur l'ensemble des diagrammes de pression différentielle nous avons reporté un diagramme théorique de référence résultant d'une poussée triangulaire $(\delta / \phi=1)$ au dessus du fond de fouille et d'une butée triangulaire sous le fond de fouille (hypothèses $\delta / \phi=0$ et -1 ). A ce diagramme est rajoutée une droite de butée théorique en tête permettant de situer l'influence de l'effet de voûte ou de la précontrainte des butons.

Dès $30 \mathrm{~cm}$ d'excavation la faible raideur du buton autorisant des déplacements non négligeables en tête conduit à un diagramme de poussée sensiblement égal au diagramme théorique (pour $\delta / \phi=2 / 3$ ). Le

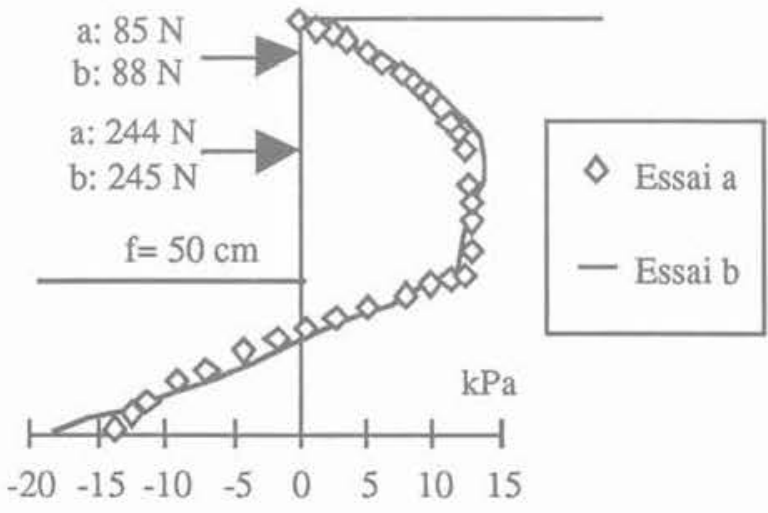

Courbes de pression différentielle

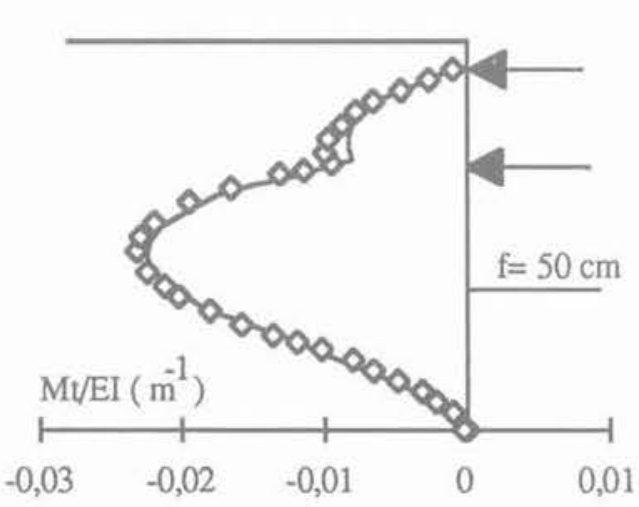

Courbes de moment fléchissant

Fig. 3. - Reproductibilité des essais.

Fig. 3. - Reproductibility of experiments. 


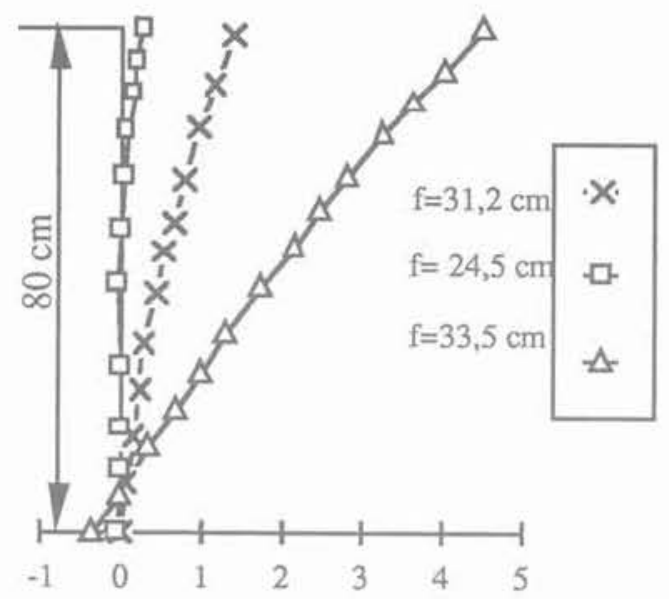

Déplacement $\Delta$ en $\mathrm{cm}$

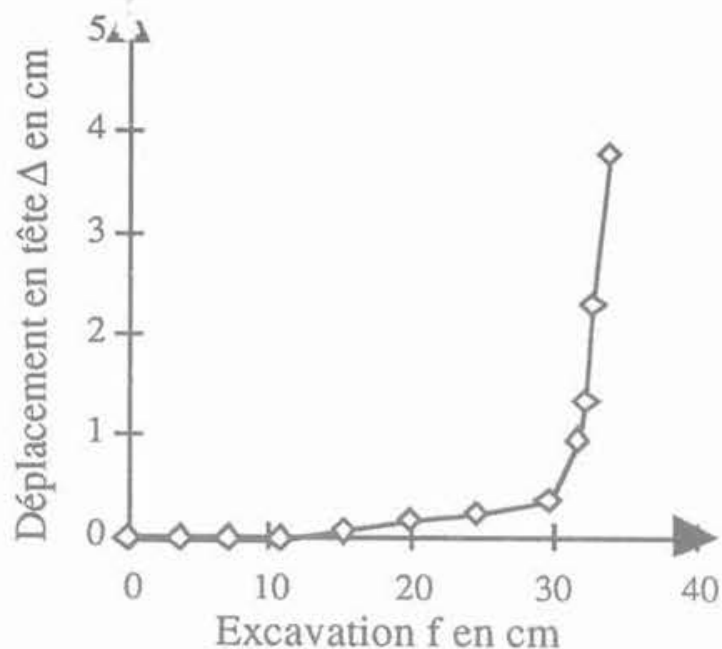

Excavation $\mathrm{f}$ en $\mathrm{cm}$

Fig. 4. - Evolution de la déformée et du déplacement en tête du rideau en fonction de l'excavation. Fig. 4. - Evolution of defiection and displacement of the top of the sheet pile relative to excavcation.
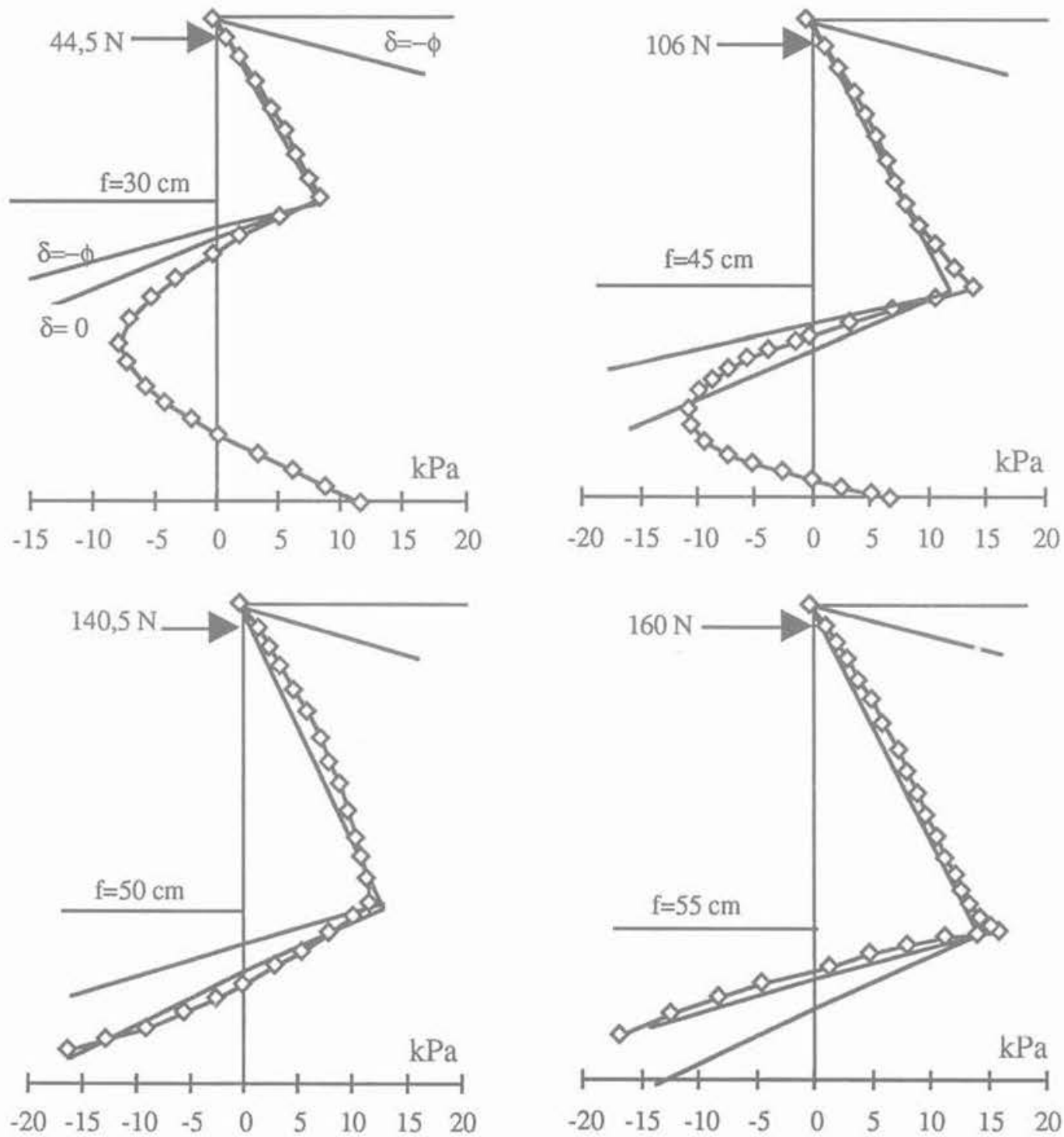

Fig. 5. - Essai 186 (1 buton déformable non précontraint). Evolution des pressions différentielles. Fig. 5. - Experiment $1 B 6$ (1 deformable non-prestressed strut). Evolution of differential earth pressure. 
déplacement relatif en tête $\Delta / \mathrm{h}$ est alors de $3 / 1000^{e}$. Pour le rideau en service $(\mathrm{h}=45 \mathrm{~cm})$, on note un comportement du type ancré encastré avec de la contre-butée au pied du rideau. Ensuite, on évolue vers un schéma de type ancré simplement buté avec un diagramme de pression passive de RANKINE pour $\mathrm{h}=50 \mathrm{~cm}(\delta / \phi=0)$, puis une mobilisation complète de la butée au voisinage de la rupture $(\mathrm{h}=55 \mathrm{~cm} ;-1<\delta / \phi<-2 / 3)$. Ces résultats sont semblables à ceux décrits par ROWE (1955).

\subsubsection{Rideau avec 1 buton rigide non précontraint} (fig. 6)

La différence essentielle par rapport à l'exemple précédent est la présence d'une concentration de pression importante au droit du butonnage, concentration qui subsiste avec la progression de l'excavation et que l'on peut rattacher à ce que l'on appelle généralement "l'effet de voute *.

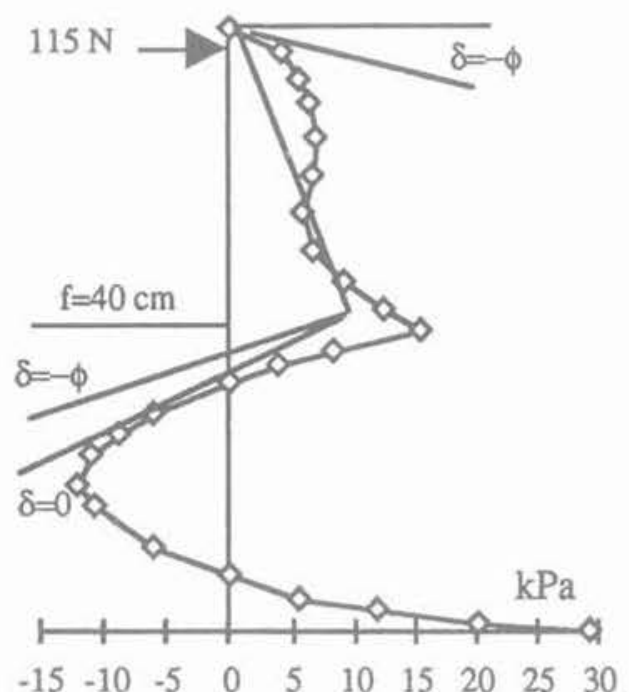

Il s'agit en effet d'une modification du diagramme de pression liée à la déformation différentielle du rideau. Le « ventre * du rideau provoque cette redistribution de contrainte avec augmentation au niveau du buton où le déplacement est pourtant quasi-nul. Il convient de remarquer que cet effet persiste pour une raideur de buton intermédiaire alors que le rideau s'écarte du massif, le déplacement relatif en tête $\Delta / h$ atteignant $4 / 1000^{e}$.

D'après ROWE (1955) cet effet disparaît dès que $\Delta / h=1 / 1000$; toutefois son mode opératoire est plus artificiel: le buton très rigide était relâché après excavation jusqu'à disparition de l'effet de voute. Dans nos essais, la déformation du rideau et le déplacement en tête sont simultanés, ce dernier étant lié au raccourcissement élastique de buton sous la charge.

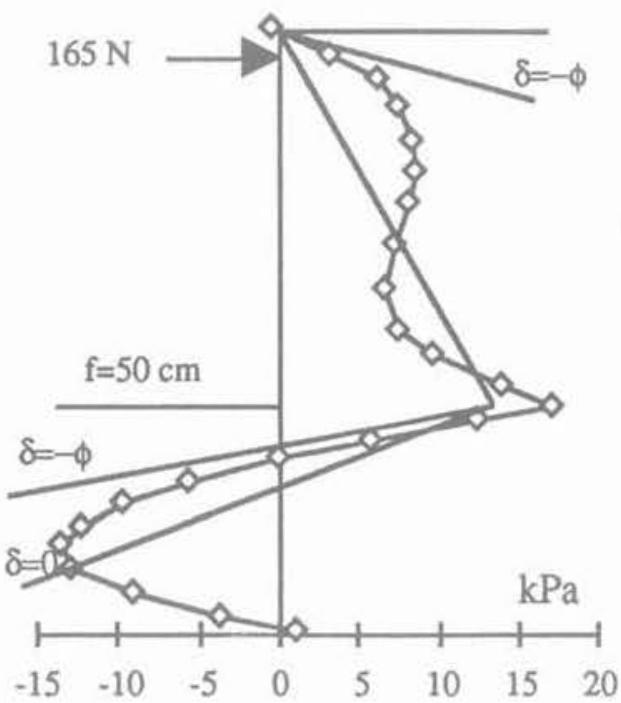

Pressions différentielles
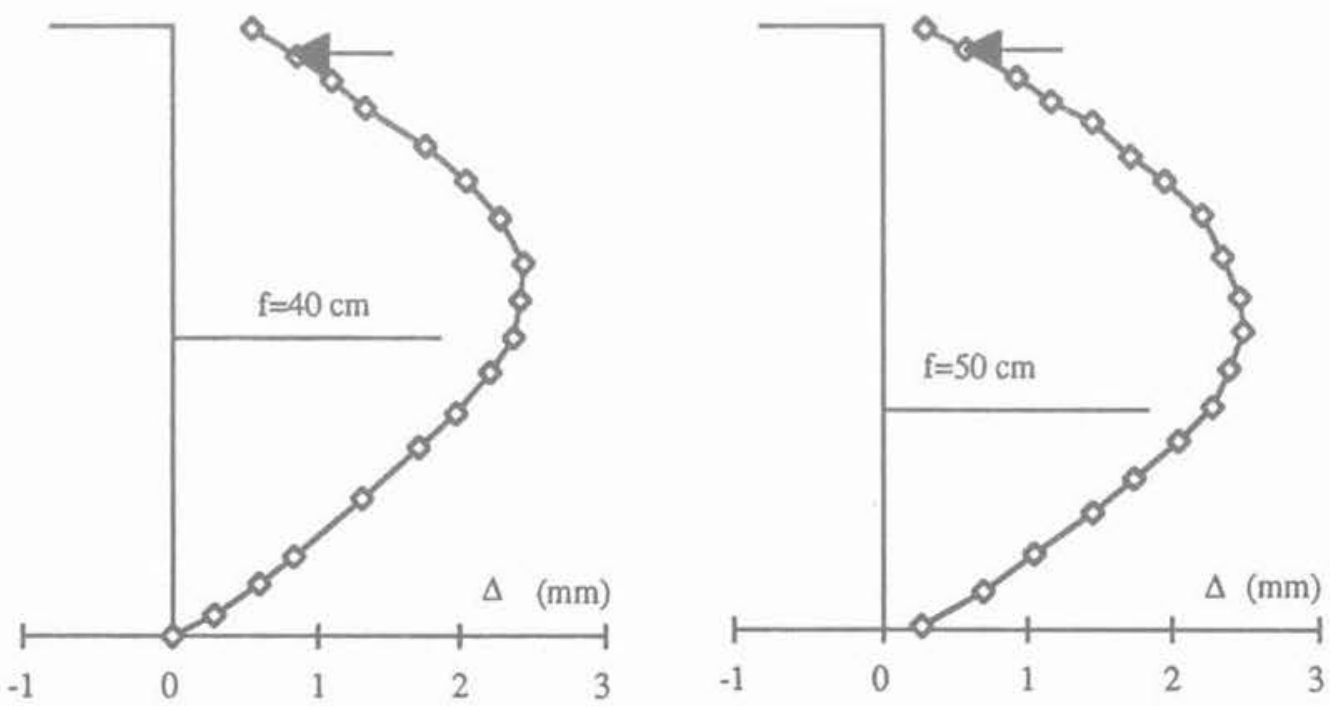

Déplacements

Fig. 6. - Rideau maintenu par un buton indéformable non précontraint. - Fig. 6. - Sheet pile with 1 non-deformable non-prestressed strut. 


\subsubsection{Rideau avec 1 buton déformable précontraint}

La précontrainte a pour objectif de limiter le déplacement des rideaux lié au raccourcissement élastique des butons.

La figure 7 présente un essai avec un buton très déformable précontraint, dès sa mise en œuvre, par un effort proche de celui attendu pour l'ouvrage en service.

Cet effort important met en butée le sol à l'arrière du rideau $(\mathrm{h}=20 \mathrm{~cm})$. Pour l'ouvrage en service, cette butée subsiste sur les 10 premiers centimètres et est prolongée par un diagramme sensiblement rectangulaire jusqu'au fond de fouille. Enfin, au voisinage de la rupture $(\mathrm{h}=55 \mathrm{~cm})$, une concentration de contrainte semblable à l'effet de voûte subsiste en tête. Son amplitude dépend du niveau de précontrainte.

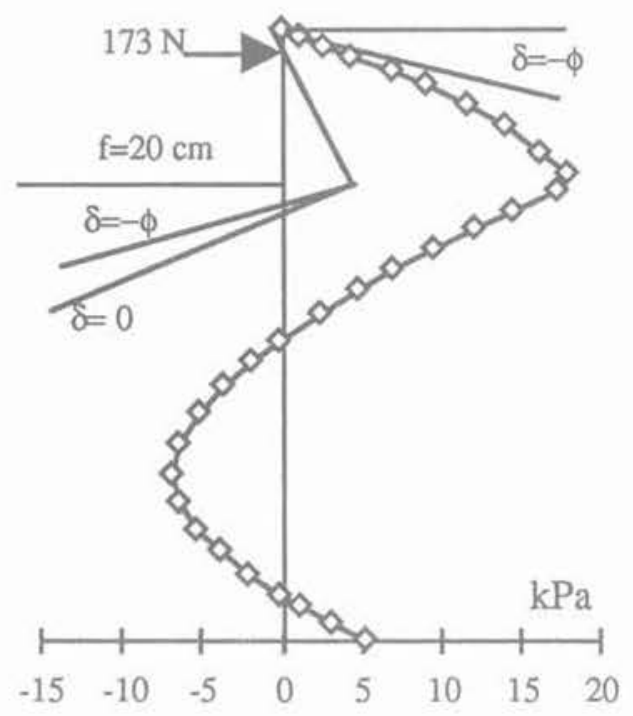

\subsubsection{Commentaires sur l'ensemble des essais avec un buton}

\section{a. Déplacement du rideau}

La figure 8 montre l'influence sensible de la raideur d'un bouton non précontraint sur le déplacement global du rideau. On note par contre que cette raideur n'a pratiquement plus d'influence dès lors que le buton est fortement précontraint. Si l'on excepte l'essai avec un buton fortement déformable et non précontraint, l'évolution des déplacements du pied du rideau en fonction de l'excavation montre que les déplacements restent faibles jusqu'à $45 \mathrm{~cm}$ d'excavation pour s'accélérer ensuite brutalement jusqu'à la rupture par manque de fiche. Ceci justifie la règle de calcul de l'ouvrage avec un coefficient de sécurité de 2 sur la butée qui correspond au seuil d'apparition des déplacements importants en pied.

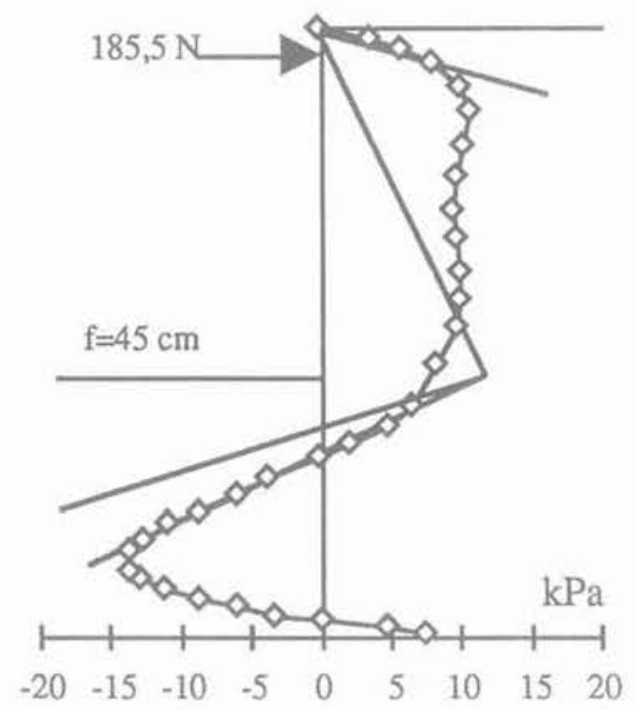

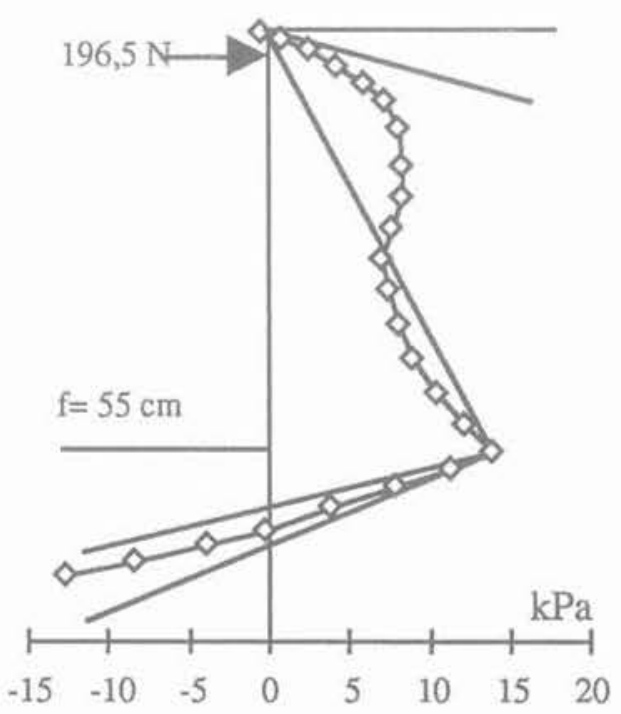

Fig. 7. - Evolution des pressions différentielles, Essai 188 (1 buton déformable et précontraint). Fig. 7. - Evolution of differetial pressures $(1$ deformable prestressed strist). 


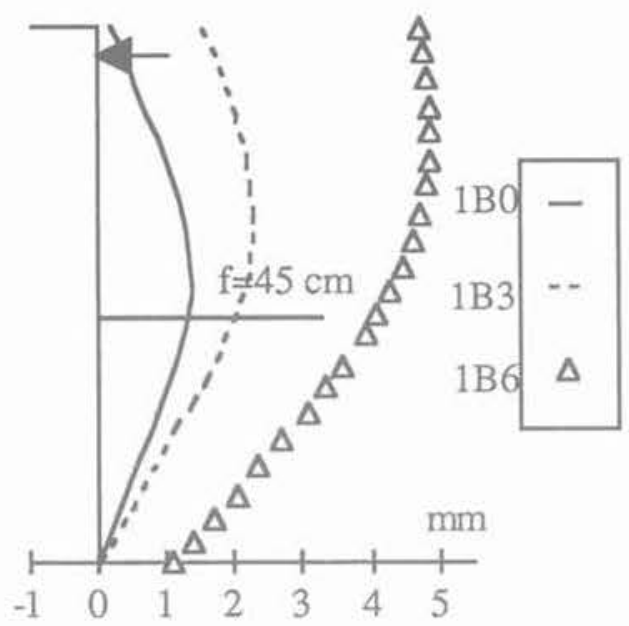

1 buton sans précontrainte

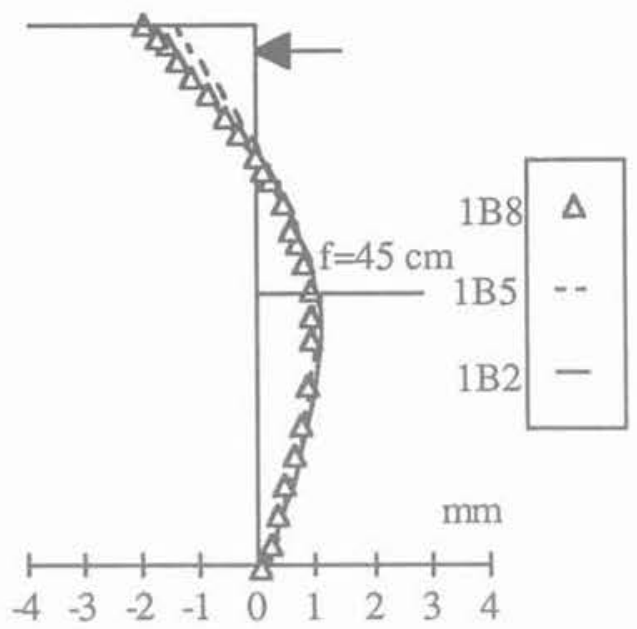

1 buton avec forte précontrainte

Fig. 8. - Courbes de déformée.

Fig. 8. - Deflection curves.

\section{b. Effort dans le buton (fig. 9)}

En l'absence de précontrainte, la valeur de l'effort augmente fortement avec la raideur du buton, l'écart atteignant $35 \%$ pour l'ouvrage en service. Cette augmentation est due essentiellement à l'effet de voûte qui provoque une concentration de contrainte au droit de lappui.

Une précontrainte élevée atténue fortement l'influence de la raideur sur l'effort, la concentration de pression au droit de l'appui étant liée pour l'essentiel à la mise en butée du sol lors de la pose du buton précontraint. c. Moment fléchissant (fig. 10)

Il est affecté simultanément par la raideur et la précontrainte du rideau et atteint la valeur du calcul en "simplement buté " dans le cas du buton rigide avec forte précontrainte.

Avec un buton très déformable non précontraint, le moment maximum chute de $30 \%$. Cette variation est toutefois bien inférieure à celle de l'effort dans le buton ( $45 \%$ ) dont l'effet sur le moment est partiellement compensé par la concentration de poussée côté terre.

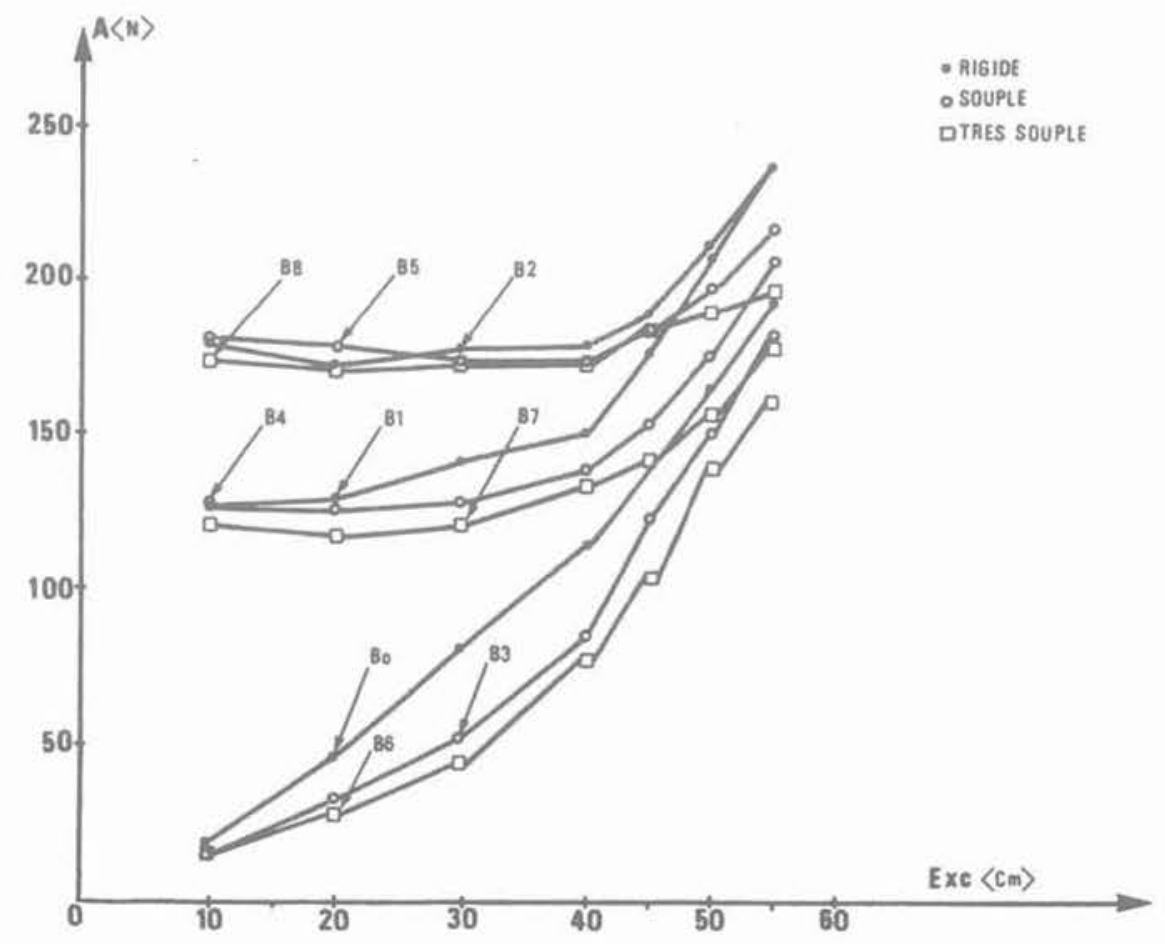

Fig. 9. - Effort dans le buton, en fonction de la hauteur d'excavation. Fig. 9. - Evolution of the strut load during excavation. 


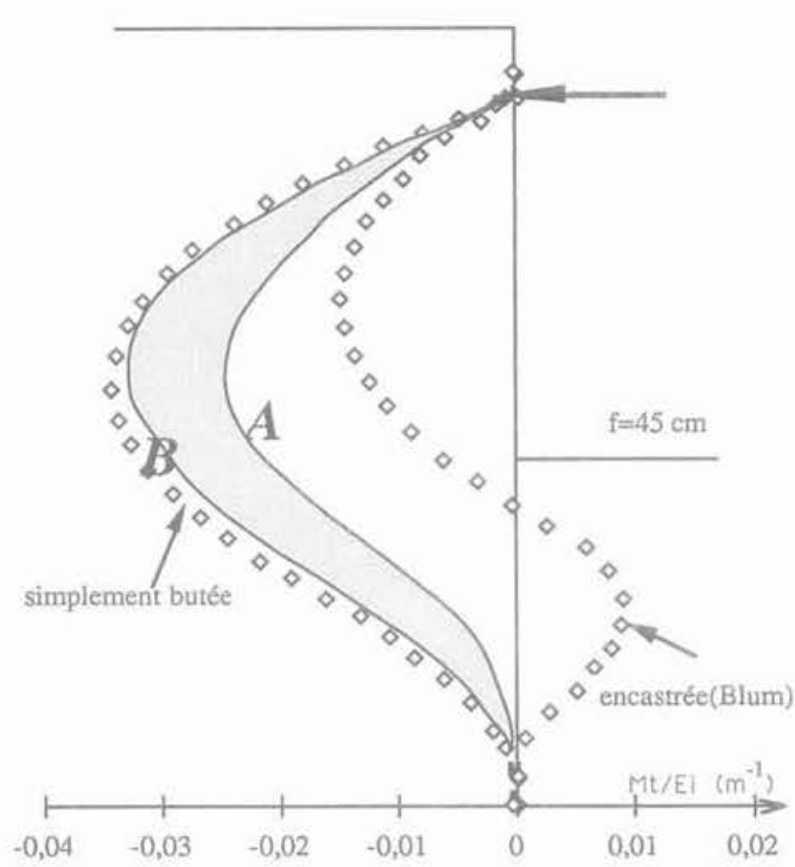

A : 1 buton très déformable sans précontrainte.

B: 1 buton rigide avec forte précontrainte.

Fig. 10. - Comparaison des courbes de moment fléchissant mesurées et celles obtenues par des calculs classiques.

Fig. 10. - Comparison of calculated and measured bending moment distribution.

Globalement pour l'ouvrage en service, le calcul suivant la méthode du rideau ancré simplement buté donne une borne supérieure du diagramme de moment fléchissant. Par contre, pour ces rideaux assez peu flexibles, la méthode de BLUM conduit à une forte sous-estimation du moment maximum.

\section{RIDEAU ANCRÉ}

Une vingtaine d'expériences sur le rideau maintenu par un ancrage ont été réalisées pour tester l'influence de la longueur, de l'inclinaison et de la précontrainte de lancrage. L'ancrage est peu déformable par luimême, mais le déplacement du corps d'ancrage sous l'effort varie avec sa longueur et son inclinaison, jouant ainsi un rôle analogue à la raideur du buton.

Globalement, le comportement du rideau ancré est identique à celui du rideau butonné, à l'exception de deux points :

- pour les ancrages non précontraints, on note une concentration de pression à l'arrière du rideau plus marquée, explicable par le développement d'un effet de voute entre le corps d'ancrage et le point d'ancrage ;

- pour des ancrages de longueur inférieure à $0,85 \mathrm{~m}$. la rupture survient par basculement de l'ensemble rideau-ancrage avant la rupture habituelle par manque de fiche, C'est la stabilité d'ensemble du dispositif de soutènement qui n'est pas assurée, comme le montre la figure 11. L'étude de cette stabilité sur la base de nos expériences fera l'objet d'une publication ultérieure.

\section{RIDEAUX MAINTENUS PAR DEUX NIVEAUX DE BUTONS}

Ce dispositif est fréquent dans les travaux urbains où l'on met en œuvre plusieurs niveaux d'étaiement afin de limiter au mieux le déplacement des soutènements. Les essais sur modèle ont été réalisés avec deux butons rigides ou déformables, précontraints ou non.

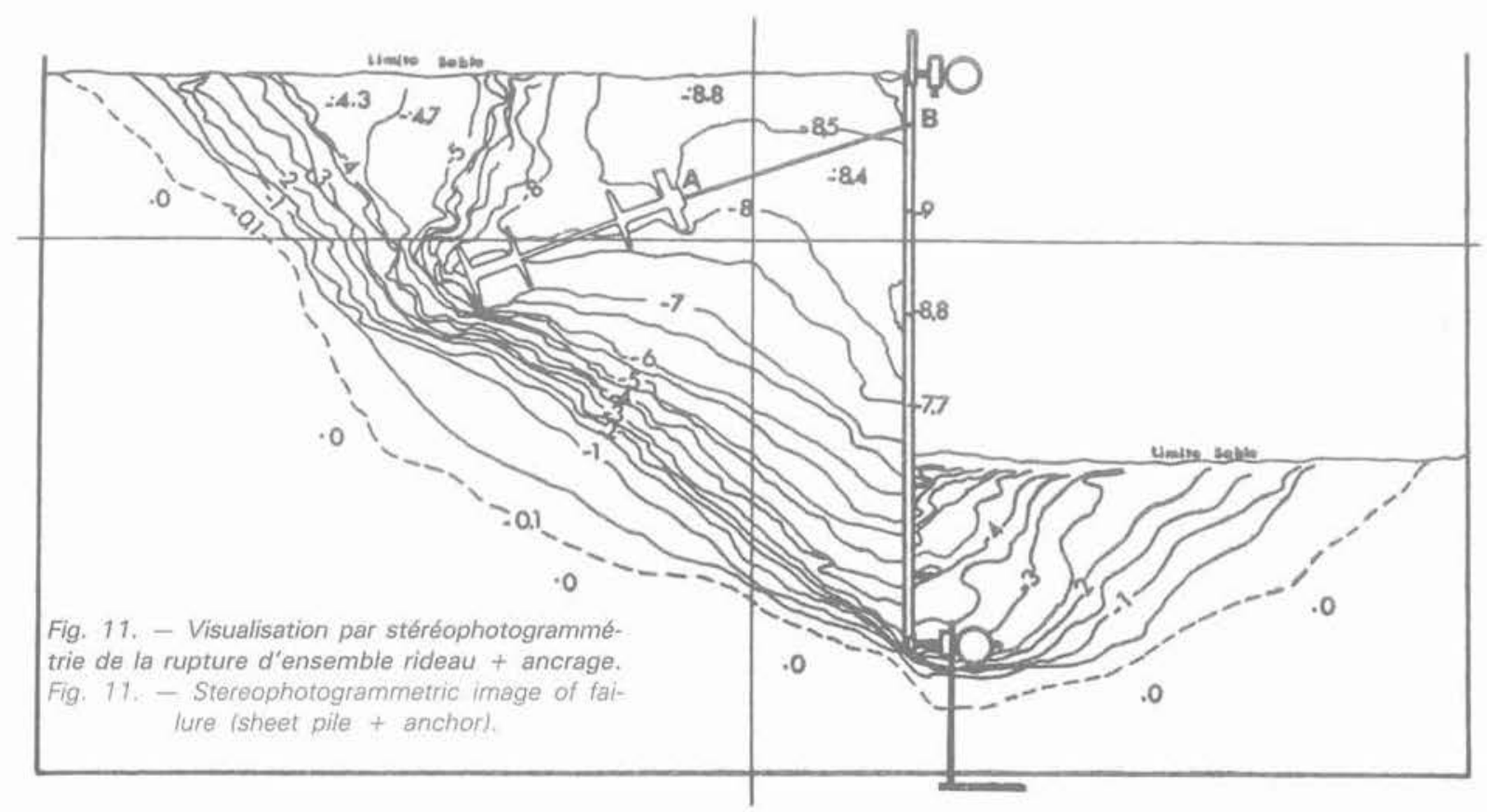

Mouvements horizontaux en (mm). 
Les résultats obtenus sont commentés en référence aux essais avec un seul buton.

\subsection{Description des essais}

Après une excavation de $15 \mathrm{~cm}$, le premier buton est posé à $5 \mathrm{~cm}$ de profondeur. On excave ensuite jusqu'à $40 \mathrm{~cm}$ pour poser le deuxième buton à $25 \mathrm{~cm}$ avant de poursuivre les terrassements par passes de $5 \mathrm{~cm}$ jusqu'à la rupture $(\mathrm{h}=63 \mathrm{~cm})$.

\subsection{Poussée à l'arrière de l'écran}

Les essais mettent en évidence de manière nette les reports de pression dus au déplacement différentiel de l'écran. Dans le cas des butons déformables non précontraints (fig. 12a) la poussée reste proche de sa valeur théorique jusqu'à $45 \mathrm{~cm}$ d'excavation. Au-delà, on note un accroissement de la poussée dans la partie supérieure, alors même que cette partie du rideau s'écarte légèrement du massif. Ce comportement s'explique par la différence de déplacement entre la tête et le pied: lorsque l'excavation passe de 45 à $60 \mathrm{~cm}$, le mouvement du pied vers la fouille est de $3 \mathrm{~mm}$ alors qu'il est inférieur à $1 \mathrm{~mm}$ en tête, créant ainsi un «point dur " relatif. La mise en place de butons peu déformables, bloquant ainsi complètement la partie supérieure du rideau, accentue nettement le phénomène comme l'indiquent les diagrammes de poussée de la figure $12 \mathrm{~b}$.

\subsection{Moment fléchissant et effort dans les butons}

Contrairement au rideau maintenu par un seul buton, la déformabilité et la précontrainte ont une influence non négligeable sur le diagramme de moment fléchissant. Ainsi, la déformabilité de l'étaiement permet de réduire les efforts dans les butons et d'améliorer leur répartition avec en contre-partie une augmentation du moment maximum de $25 \%$.

La précontrainte des butons s'avère efficace en permettant, au prix d'une augmentation d'effort, de réduire les déplacements du rideau ainsi que le moment fléchissant maximum.

\subsection{Comparaison avec les diagrammes empiriques de TERZAGHI et PECK (1965)}

En l'absence de précontrainte, ces diagrammes restent une enveloppe supérieure de la poussée du sol dans la partie hors fiche, tout au long des terrassements (fig. 13).

Par contre, pour les butons précontraints, ce diagramme sous-estime la pression du sol, en particulier lorsque la fiche est importante.

\section{CALCULS PAR LA MÉTHODE DU MODULE DE RÉACTION}

Le module de réaction $\mathrm{K}_{\mathrm{h}}$ n'étant pas une caractéristique intrinsèque du sol, la définitiion de la loi de réaction varie avec les auteurs, en particulier dans le cas des soutènements où il faut compléter $K_{h}$ par des seuils de pression active et passive et par la prise en compte des irréversibilités de comportement. Sur le même principe du module de réaction, on trouve ainsi divers logiciels plus ou moins proches en fonction des hypothèses complémentaires adoptées. Pour cette étude, nous avons utilisé le programme RIDO développé à l'occasion de la construction du métro de Lyon.

Le principe des calculs de palplanches par la méthode du module de réaction ayant été abondamment décrit nous présenterons ici uniquement le principe de la loi de réaction adoptée dans le programme RIDO.

\subsection{Loi de réaction adoptée dans « RIDO »}

Dans ce logiciel, la pression est prise en compte séparément de part et d'autre du rideau. A Porigine, elle est égale à la pression des terres au repos, avec prise en compte de l'effet de surcharges éventuelles. En un point, elle varie ensuite linéairement avec le déplacement horizontal $\mathrm{Y}$, tout en étant limitée par les valeurs correspondant à la poussée ou à la butée.

Cette loi de réaction s'écrit :

$$
P=P_{0}+K_{h} \cdot(y-v)
$$

avec :

$P_{0}$ : pression des terres au repos

$y$ : déplacement horizontal du rideau au point considéré

$v$ : facteur rendant compte de l'hystérésis

$\mathrm{K}_{\mathrm{h}}$ : module de réaction défini par la relation

où :

$$
\mathrm{K}_{\mathrm{h}}=\mathrm{R}_{e}+\mathrm{R}_{\mathrm{p}} \cdot \sigma_{\mathrm{v}}
$$

$\mathrm{R}_{e}\left(\right.$ en $\left.\mathrm{kN} / \mathrm{m}^{3}\right)$ : partie constante du module de réaction ;

$\mathrm{Rp}\left(\right.$ en $\mathrm{m}^{-1}$ ) : coefficient d'augmentation de $\mathrm{K}_{\mathrm{h}}$ avec la contrainte verticale effective $\sigma_{v}$ au point considéré.

\subsubsection{Irréversibilité}

Si à la fin d'une phase $n$, une des limites de plasticité est atteinte (poussée ou butée), v est recalculé pour conduire à la nouvelle loi de réaction conformément à la figure 14 pour la phase $n+1$.

\subsubsection{Variation de contrainte verticale}

Lorsque la contrainte verticale varie (en cas d'excavation, de remblaiement ou de surcharge par exemple) divers choix sont possibles pour redéfinir le couple $\mathrm{p}_{\mathrm{o}}-\mathrm{v}$. Le choix fait dans RIDO est de conserver alors la même valeur de v (fig. 15),

\subsection{Choix des paramètres pour la simulation des essais sur modèle.}

\section{a. Coefficients de pression du sol}

La pression au repos (Ko) du matériau analogique a été déterminée sur un modèle de poussée-butée. Sa valeur, égale à 0,9 est plus élevée que celle donnée par la relation de Jacky (Ko $\approx 1-\sin \phi=0,64$ ) ce qui peut s'expliquer par le caractère bidimensionnel de matériau. Elle correspond au calcul en élasticité : pour un matériau bidimensionnel $\mathrm{Ko}=$ coeffi- 

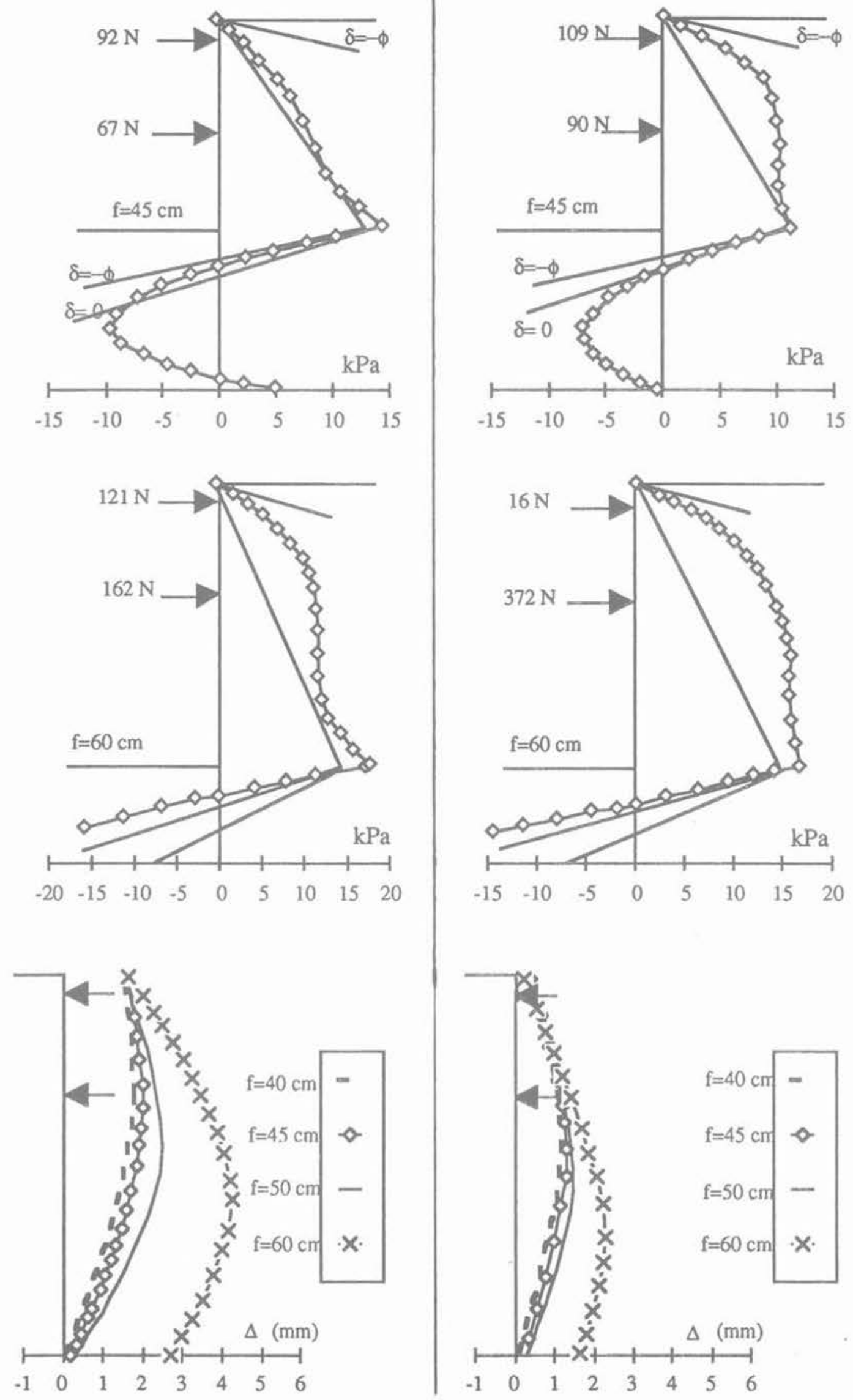

Fig. 12a. -2 butons souples non précontraints. Fig. 12a. -2 deformable non-prestressed struts.

Fig. 12b. -2 butons rigides non précontraints. Fig. 12b. -2 non-deformable non-prestressed strusts. 

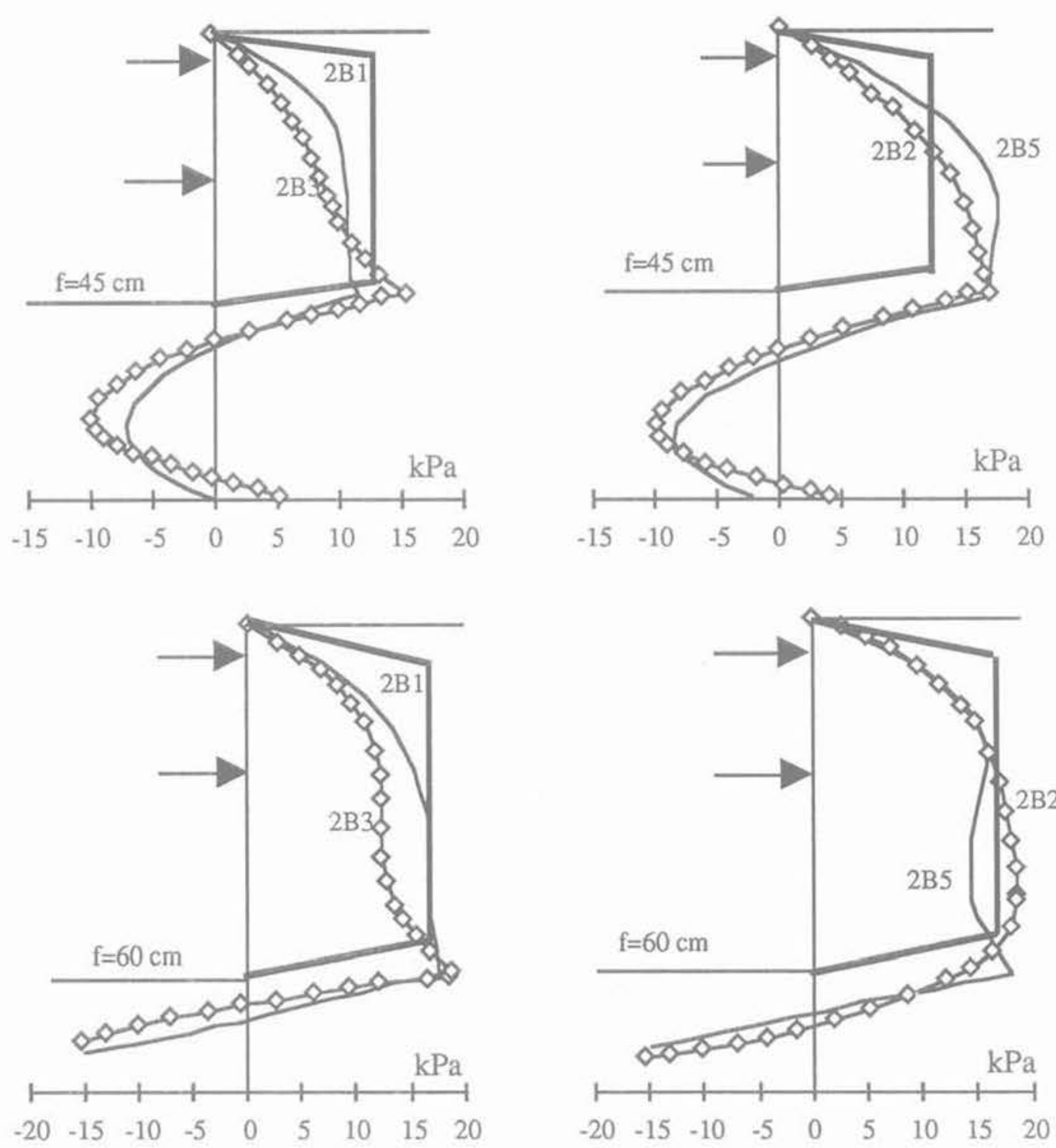

Fig. 13. - Comparaison des poussées mesurées avec le diagramme proposé par TERZAGHI et PECK (2B1 et 2B3: non-précontraints $2 B 2$ et 2B5: précontraints).

Fig. 13. - Comparison of measured earth pressure with the diagram proposed by TERZAGHI and PECK.

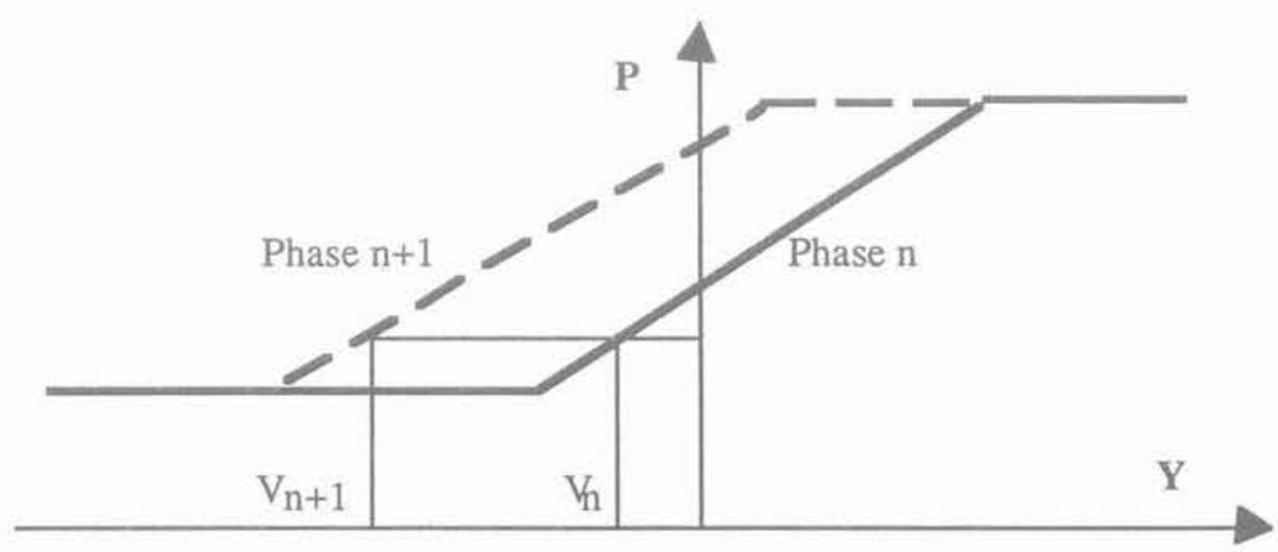

Fig. 14. - Loi de réaction du programme RIDO et son comportement irréversible. Fig. 14. - Reaction law of RIDO program and its irreversibility. 


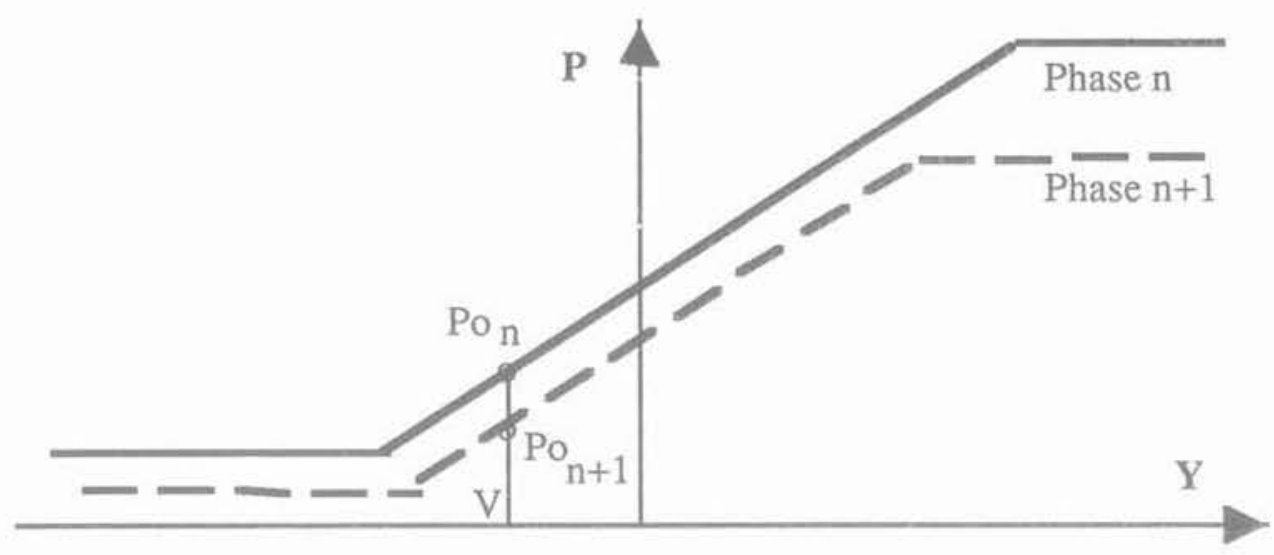

Fig. 15. - Evolution de la loi de réaction avec la contrainte verticale.

Fig. 15 - Influence of vertical stress on reaction law.

cient de Poisson $v$ dont la valeur déduite des essais biaxiaux est voisine de 0,9 .

Pour les coefficients de pression active et passive, on a admis que le frottement maximum sol-écran pouvait être mobilisé pour de forts déplacements et adopté les valeurs théoriques correspondantes, soit pour $\phi=21^{\circ}$ et $|\delta / \phi|=1$ :

$$
\begin{aligned}
& \mathrm{K}_{\mathrm{a \gamma}} \cos \delta=0,39 \\
& \mathrm{~K}_{\mathrm{p \gamma}} \cos \delta=3,1
\end{aligned}
$$

\section{b. Module de réaction}

Le module étant un paramètre de calcul et non un paramètre intrinsèque du sol, il n'est pas directement mesurable. Pour un même sol, il peut varier avec la géométrie et la nature des mouvements de l'écran.

En pratique, on utilise des valeurs empiriques ou déduites d'essais tels que l'essai pressiométrique (BALAY, 1985).

En l'absence de base empirique pour ce matériau, une rétro-analyse des deux premiers essais a été effectuée, conduisant à une valeur du module proportionnelle à la contrainte verticale, soit :

avec

$$
\begin{aligned}
& \mathrm{K}_{\mathrm{h}}=\mathrm{R}_{\mathrm{p}} \cdot \sigma_{\mathrm{v}} \\
& \mathrm{R}_{\mathrm{p}}=1500 \mathrm{~m}^{-1}
\end{aligned}
$$

Par la suite, cette valeur a été conservée pour l'ensemble des simulations numériques.

\section{COMPARAISONS CALCULS-MESURES}

Tous les essais ont fait l'objet d'un calcul avec le même jeu de paramètres afin de comparer les courbes de moment fléchissant, de pression différentielle, de déplacement ainsi que les efforts de butonnage ou d'ancrage. L'analyse de ces résultats pour chaque type de rideau testé en référence à l'hypothèse du module de réaction, permet, outre la mise en évidence des limites de ces calculs, de situer l'origine des écarts constatés.

\subsection{Rideau non ancré}

C'est la rotation du rideau par rapport à sa base qui caractérise cet essai. Pour le rideau en service, les déplacements calculés et mesurés sont voisins. Notons toutefois que les déplacements calculés sont ici, en l'absence de butons, fortement contrôlés par la valeur du module de réaction, choisie a posteriori (fig. 16). Si l'on examine plus précisément la déformée, on note que l'encastrement réel est mal traduit par le calcul.

Pour le moment fléchissant, le calcul satisfaisant audessus du fond de fouille sous-estime fortement la valeur en dessous (fig. 17).

Ces constatations indiquent que:

- la pression active du matériau de SCHNEEBELI est proche de sa valeur théorique;

- dans le calcul, la butée maximale est mobilisée trop rapidement sous le fond de fouille : un schéma de réaction complexe, proche de courbes de butée classiquement mesurées sur modèles par divers auteurs, devrait permettre de corriger en partie cet écart ;

- la contre butée qui est à l'origine de l'encastrement du pied de rideau, est sous-estimée par le calcul. Ce résultat n'est pas surprenant, l'expérimentation indiquant que la contre butée peut se développer en l'absence de déplacement du pied de l'écran qui joue alors le rôle d'un « point dur » par rapport au sommet du rideau incurvé vers la fouille. $\mathrm{Ce}$ phénomène du type effet de voûte, ne peut être déduit par l'hypothèse de module de réaction qui lie les changements des pressions du sol en un point au déplacement de ce seul point.

\subsection{Rideaux maintenus par un niveau de butons et d'ancrages}

Le déplacement de ces rideaux est contrôlé, en tête par le butonnage et en pied par le sol en butée. Pour l'excavation correspondant au rideau " en service " ces déplacements restant très faibles, les déplacements calculés et mesurés restent proches. Au-delà, lorsque l'on poursuit l'excavation, le calcul des déplacements n'est 

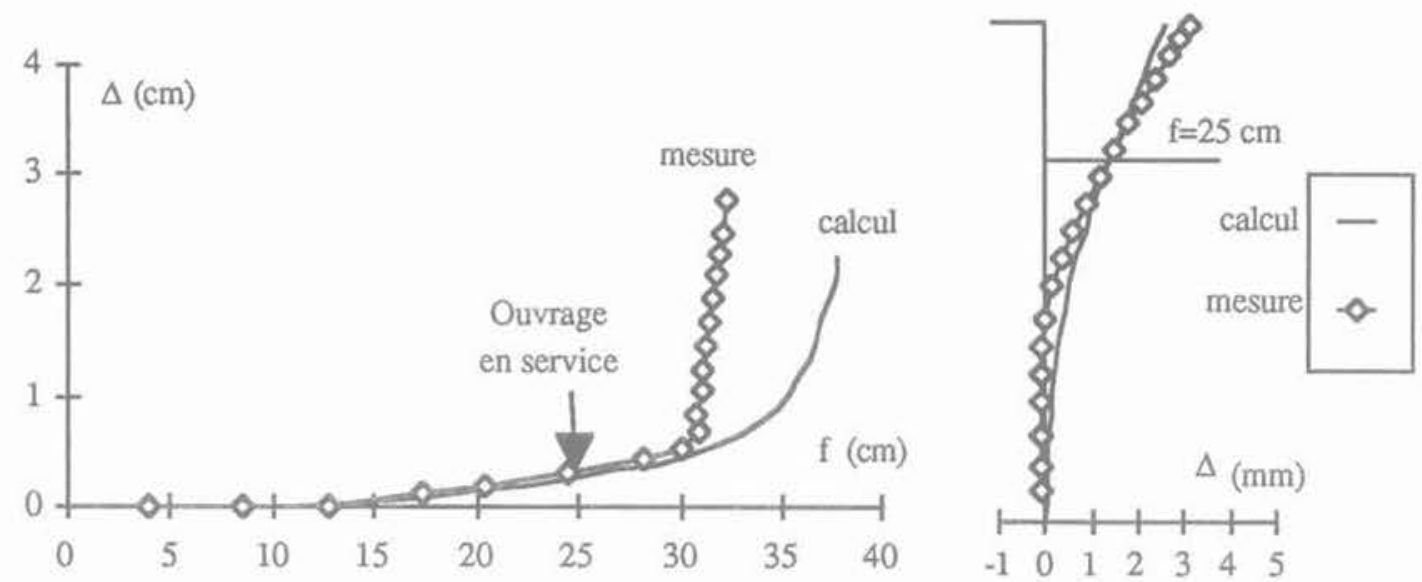

Fig. 16. - Rideau non butonné. Comparaison des déplacements mesurés et calculés.

Fig. 16. - Sheet pile without any strut. Comparison of calculated values of displacement to measured values.
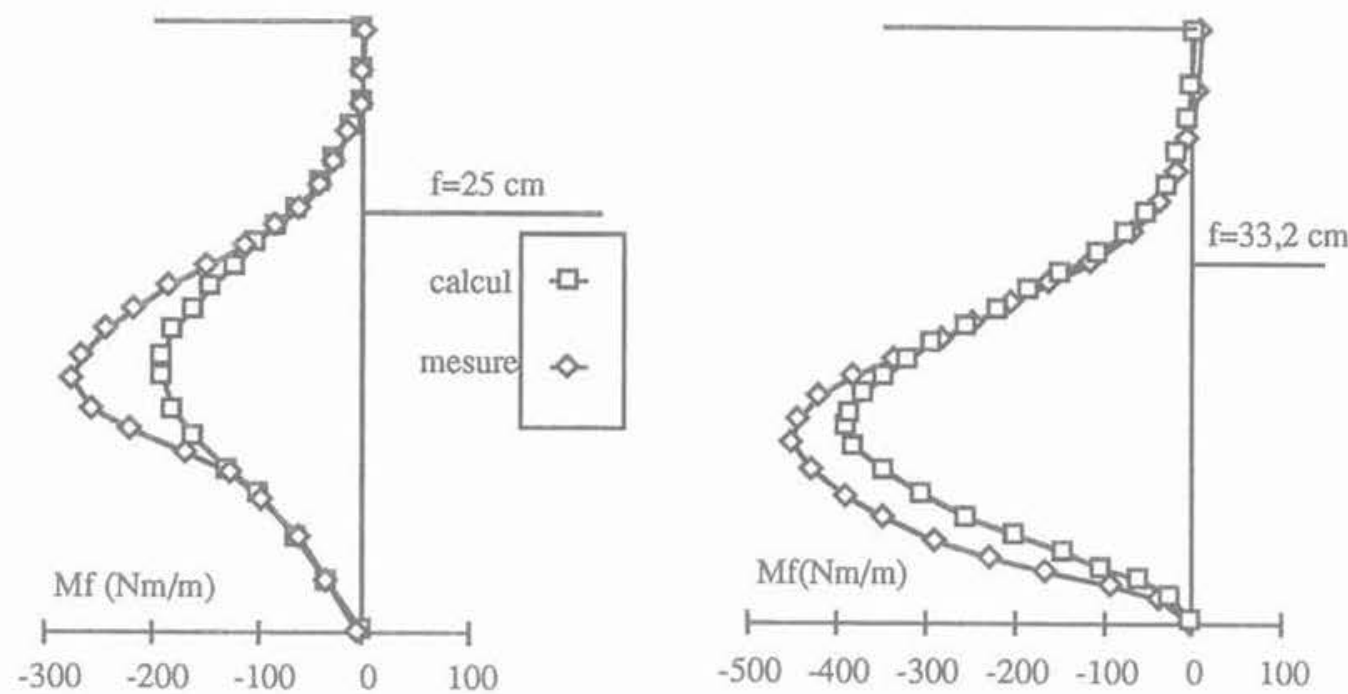

Fig. 17. - Rideau non butonné. Comparaison des moments fiéchissants mesurés et calculés.

Fig. 17. - Sheet pile without any strut. Comparison of calculated values of bending moment to measured values.

plus significatif ; par contre il donne une valeur correcte de la fiche correspondante à la rupture par manque de butée.

\subsubsection{Butons non précontraints}

\section{a. Buton très déformable}

Cette déformabilité autorisant une rotation non négligeable du rideau par rapport à la base, on retrouve partiellement les particularités du rideau non ancré :

le calcul surestimant la butée en fond de fouille, il en résulte une sous-estimation de l'effort dans le buton et du moment fléchissant atteignant $20 \%$ (fig. 18).

\section{b. Buton non déformable}

La répartition de pression calculée à l'arrière du rídeau est triangulaire, l'hypothèse de WINKLER ne pouvant traduire la concentration de poussée due à l'effet de voûte. En conséquence, l'effort dans le buton est sous-estimé de plus de $30 \%$. Paradoxalement, les moments fléchissants calculés et mesurés sont très proches, cette concentration de poussée étant compensée par un déficit équivalent d'effort dans le buton.

\subsubsection{Buton précontraint}

Dans le calcul comme sur le modèle, la précontrainte du buton met le sol en butée à l'arrière du rideau. Les diagrammes calculés et expérimentaux sont alors très proches, que le buton soit déformable ou non : le calcul simule bien l'expérimentation pour l'ensemble des paramètres mesurés.

\subsubsection{Rideaux maintenus par un niveau d'ancrage}

Par rapport au rideau butonné, la présence de l'ancrage à l'arrière du rideau induit deux phénomènes complémentaires :

- d'une part, une augmentation de la concentration de poussée au niveau d'ancrage, provoquée par un effet de voute entre ce point et le corps d'ancrage. Il en résulte, même dans le cas des ancrages précontraints, une accentuation des écarts entre calcul et mesures pour l'effort d'ancrage ;

- d'autre part, lancrage mobilise l'ensemble du massif à l'arrière du rideau et induit un déplacement d'ensemble d'autant plus important que le tirant est court. 


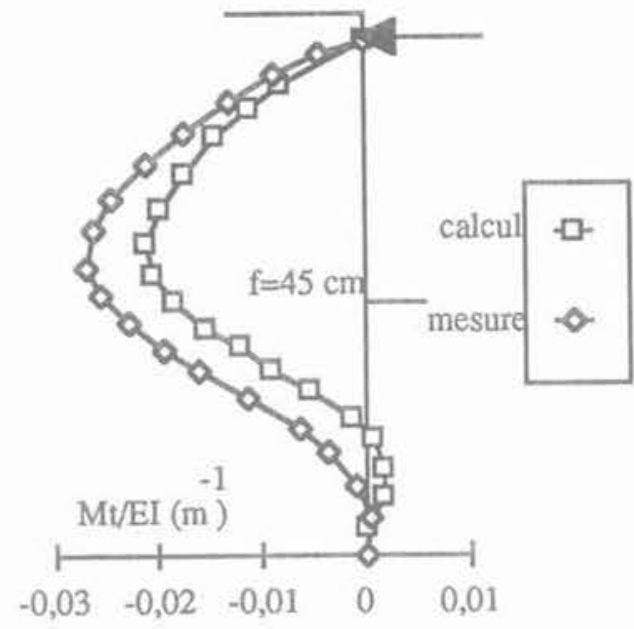

1 buton souple sans précontrainte

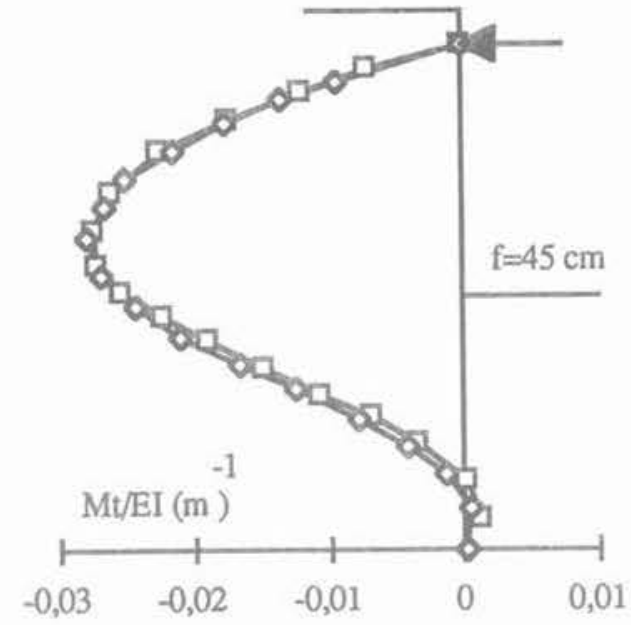

1 buton souple avec forte précontrainte

Fig. 18. - Comparaison calcul-mesure des courbes de moment fléchissant.

Fig. 18. - Comparison of calculated values of bending moment to measured values.

Le principe du calcul étant basé sur la relation locale déplacement-pression, cet effet ne peut être pris en compte.

\subsection{Rideaux maintenus par deux niveaux de butons}

\subsubsection{Butons non précontraints}

Ce sont les reports d'efforts dus aux déformations différentielles qui caractérisent ces essais. L'hypothèse de WINKLER étant incapable de traduire correctement ces reports, on constate logiquement des écarts importants entre calculs et mesures, le calcul sous-estimant systématiquement la poussée à l'arrière du rideau, l'effort dans les butons et le moment fléchissant maximum. Ainsi, à titre d'exemple, avec les butons rigides le programme de calcul sous-estime l'effort global dans les butons de $36,5 \%$ et le moment fléchissant maximum de $28 \%$. Par contre, le déplacement global du rideau est correctement évalué.

\subsubsection{Butons précontraints}

Bien que les diagrammes de pression aient la même allure qu'avec les butons non précontraints, la valeur élevée de la pression à l'arrière du rideau est due ici à la mise en butée du sol lors de la pose des butons et leur précontrainte.

Dans ces conditions, pour $45 \mathrm{~cm}$ d'excavation on constate que le calcul au module de réaction décrit bien ces essais, le diagramme de pression différentielle comme celui de moment fléchissant étant quasi confondus (fig. 19). Pour les excavations plus importantes, ( $\mathrm{h}=55 \mathrm{~cm}$ ) lorsque le pied du rideau commence à chasser par manque de butée, on retrouve des déformations différentielles du rideau avec reports de poussée que l'hypothèse du module de réaction ne peut décrire: le calcul sous-estime alors à nouveau les efforts, moments et poussée.

Enfin, il faut noter que le calcul surestime les déplacements lors de la précontrainte des butons. Ceci con-

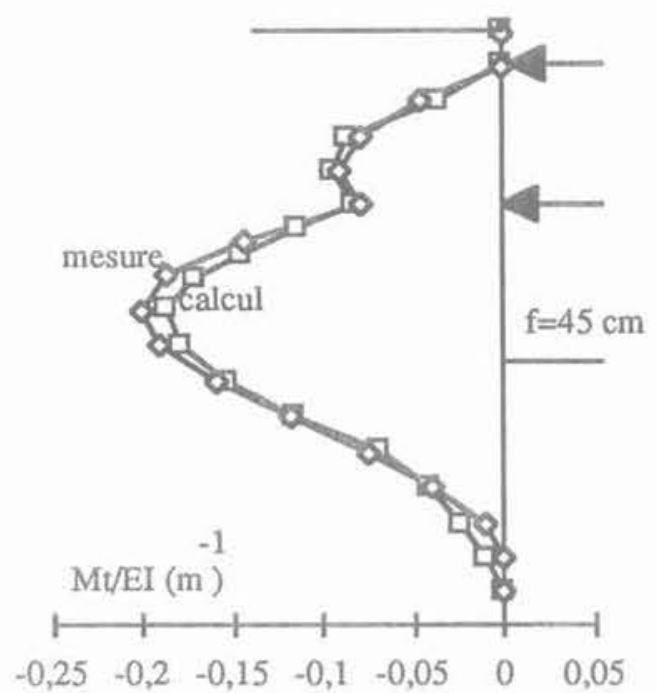

Fig, 19. - Comparaison calcul-mesure de la courbe de moment fléchissant 12 butons précontraints).

Fig. 19. - Comparison of calculated values of bending moment to measured values $(2$ prestressed struts).

firme les constatations effectuées in situ par GIGAN et qui ont conduit le LCPC à recommander une augmentation du module de réaction lors des phases de mise en tension des tirants, dans la zone concernée par cette opération.

\section{CONCLUSIONS}

Cette campagne d'essais était destinée, dans un premier temps, à cerner le comportement d'un rideau de flexibilité moyenne, tels que ceux utilisés en site urbain, dans diverses conditions d'étaiement, puis dans un deuxième temps à confronter les résultats aux méthodes de calcul. Pour les rideaux maintenus par des tirants ou butons non précontraints, ces essais 
confirment la formation d'une concentration de pression liée à l'effet de voûte au droit de l'étaiement, même pour des butons déformables. Cet effet est d'autant plus sensible que le rideau est mieux maintenu en tête comme le montrent les essais avec deux niveaux de butons

La précontrainte des butons (ou ancrages) présente deux avantages: elle limite le déplacement des rideaux et atténue fortement les effets de la déformabilité des étais. Ces avantages entraînent cependant une augmentation de l'effort dans les butons et du moment maximum.

La confrontation des essais avec un buton aux méthodes de calcul classiques en plasticité montre que, pour ces parois de flexibilité moyenne, la méthode du rideau ancré encastré sous-estime fortement le moment maximum, alors que la méthode du rideau ancré simplement buté (avec un coefficient de sécurité de 2 sur la butée) donne une valeur par excès de ce moment. Notons que la fiche obtenue avec cette dernière méthode correspond au seuil au-delà duquel les déplacements du pied du rideau deviennent importants, ce qui justifierait cette règle de calcul. Par contre, ces méthodes classiques ne sont pas adaptées au cas des butons ou ancrages précontraints qui mettent partiellement en butée le sol à l'arrière du rideau

L'hypothèse de WINKLER, utilisée dans de nombreux logiciels de calcul n'est qu'une approche très simplifiée de l'interaction sol-rideau. Ses limites apparaissent essentiellement lorsque les butons ne sont pas précontraints : les reports d'efforts dus à la déformation différentielle ne pouvant être traduits par cette hypothèse, les efforts dans l'étaiement sont sous évalués dans tous les cas. Pour certaines configurations (rideaux maintenus par un buton très déformable ou par deux butons), le moment fléchissant maximum est également sous évalué

Dès que les butons sont précontraints, les phénomènes de reports d'effort ne sont plus prépondérants et on note alors que les calculs au module de réaction traduisent correctement les efforts et moment fléchissants observés. Seuls les déplacements, lors des phases de mise en précontrainte des étais sont sous évalués ce quí confirme la nécessité d'augmenter localement $\mathrm{K}_{h}$ lors de telles phases.

\section{BIBLIOGRAPHIE}

BACOT J., KASTNER R., MEMIER A. (1984), Recherche en laboratoire de mécanique de sols. Une utilisation particulière de la photogrammétrie. Bul. SFPT, n 94,1984 , pp. 15-29.

BALAY J. (1985), Recommandations pour le choix des paramètres de calcul des écrans de soutène. ment par la méthode aux modules de réactions. LCPC, Note d'information technique.

BALAY J., HARFOUCHE L., HUMBERT P. (1985), Prédiffusion du programme PAREF, pour le cal. cul des écrans de soutènement par la méthode des éléments finis. LCPC Février 1985, 74 p.

BOISSIER D., GIELLY J., KASTNER R., MANGIN J.C. (1978), Détermination des moments et des pressions exercés sur un écran à partir de mesures inclinométriques. Revue Canadienne de Géotechnique, Vol. 15, n 4, 1978, pp. 522-536.

BOUDIER J., COLIN C., MASTIKIAN L. (1970), Calcul de stabilité des parois sur ordinateur. Travaux, Déc. 1970, pp. 40-45.

FAGES R., BOUYAT C. (1971), Modèle mathématique intégrant le comportement irréversible du sol en état élasto-plastique. Exemple d'application. Etude de l'influence des paramètres. Travaux. $n^{\circ} 441$, déc. 1971 , pp. 38-46.

GIGAN J.P. (1979), Expérimentation en vraie grandeur d'un rideau de palplanche. Revue Française de Géotechnique, n 8, 1979, pp. 27-44.

HALIBURTON T.A. (1968), Numerical analysis of flexible retaining structures. ASCE, Journal of the Soil Mechanics and Foundations Division, $n^{\circ}$ SM6, Nov. 1968, pp. 1233-1251.

KASTNER R. (1982), Excavations profondes en site urbain. Problèmes liés à la mise hors d'eau. Dimensionnement des soutènements butonnés. Thèse de Docteur ès-Science, INSA Lyon et Université Claude-Bernard, Lyon 1, 1982, 409 p.

MASROURI F. (1986), Comportement des rideaux de soutènement semi-flexibles : étude théorique et expérimentale. Thèse de Doctorat, INSA Lyon, Fév, 1986, 247 p.

MONNET J., KASTNER R., LAREAL P., BOUYAT C. (1985), Finite element calculation and experimentings on the Saxe-Gambetta station. Proceeding of the 5th International Conference on Numerical Methods in Geomechanics, Nagoya, April 1985, pp. 747-753.

ROWE P.W. (1952), Anchored sheet pile walls. Institution of Civil Engineers, Proc. Vol. 1, London, Jan. 1952, pp. 27-70.

ROWE P.W. (1955), A theorical and experimental analysis of sheet pile walls. Institution of Civil Engineers, Proc. Vol. 4, London, Jan. 1955, pp. 32-69.

ROWE P.W. (1972), Earth pressure on flexible structures. Oral presentation. $5^{e}$ Congrès Européen de Mécanique des Sols et des Travaux de Fondation, Madrid 1972

SCHNEEBELI G. (1957), Une analogie mécanique pour l'étude de la stabilité des ouvrages en terre à 2 dimensions. $4^{\mathrm{e}}$ Congrès International de Mécanique des Sols et des Travaux de Fondation, Vol. 2, Londres 1957, pp. 228-232.

TERZAGHI K., PECK R.B. (1965), Chap. VIII Pres sion des terres et stabilité des talus. Mécanique des Sols Appliquée, Dunod 1965, pp. 318-418.

WINKLER E. (1867), Die lehre von elastizitat und festigkeit. Prague, 182 p. 


\section{ANNEXE}

Tableau 1. - Essais effectués avec un niveau de buton. Table 1. - Experiments realized with one level of struts.

\begin{tabular}{|c|c|rl|}
\hline$N^{\circ}$ essai & $\begin{array}{c}\text { Précontrainte } \\
\text { (kN/m.ml) }\end{array}$ & \multicolumn{1}{|c|}{$\begin{array}{c}\text { Raideur } \\
(\mathrm{kN} / \mathrm{m} . \mathrm{ml})\end{array}$} \\
\hline 1B0 & 0,000 & 83333 rigide \\
1B1 & 2,133 & 83333 rigide \\
1B2 & 3,025 & 83333 rigide \\
1B3 & 0,208 & 816 souple \\
1B4 & 2,100 & 816 souple \\
1B5 & 3,000 & 816 souple \\
1B6 & 0,191 & 404 très souple \\
1B7 & 2,016 & 404 très souple \\
1B8 & 2,916 & 404 très souple \\
\hline
\end{tabular}

Tableau 2. - Essais effectués avec deux niveaux de buton. Table 2, - Experiments realized with 2 level of struts.

\begin{tabular}{|c|c|c|rl|}
\hline \multirow{2}{*}{$\begin{array}{c}N^{\circ} \\
\text { Essai }\end{array}$} & \multicolumn{2}{|c|}{ Précontrainte $(\mathrm{kN} / \mathrm{m} . \mathrm{ml})$} & \multirow{2}{*}{$\begin{array}{c}\text { Raideur de buton } \\
(\mathrm{kN} / \mathrm{m} . \mathrm{ml})\end{array}$} \\
\cline { 2 - 3 } & Buton 1 & Buton 2 & & \\
\hline 2B1 & 0,383 & 0,333 & 83333 & rigides \\
2B2 & 1,333 & 2,691 & 83333 & rigides \\
2B3 & 0,366 & 0,400 & 816 & souples \\
2B4 & 2,075 & 2,700 & 816 & souples \\
2B5 & 1,733 & 4,041 & 816 & souples \\
\hline
\end{tabular}

Tableau 3. - Essais effectués avec un tirant.

Table 3. - Experiments realized with one anchor.

\begin{tabular}{|c|c|c|c|}
\hline$N^{\circ}$ essai & $\begin{array}{c}\text { Longueur } \\
\text { totale }(\mathrm{m})\end{array}$ & Inclinaison & $\begin{array}{c}\text { Précontrainte } \\
\text { (kN/m.ml) }\end{array}$ \\
\hline A1 & 1,05 & 39 & 0,770 \\
A2 & 1,05 & 39 & 2,848 \\
A3 & 1,17 & 22 & 0,605 \\
A4 & 1,17 & 22 & 2,656 \\
A5 & 1,08 & 31 & 0,880 \\
A6 & 1,08 & 31 & 2,800 \\
A7 & 1,14 & 45 & 0,635 \\
A8 & 1,14 & 45 & 3,068 \\
A9 & 1,17 & 39 & 0,376 \\
A10 & 1,17 & 39 & 3,253 \\
A11 & 1,14 & 30 & 0,360 \\
A12 & 1,14 & 30 & 2,908 \\
A13 & 1,05 & 22 & 0,566 \\
A14 & 0,95 & 22 & 0,647 \\
A15 & 0,85 & 22 & 0,208 \\
A16 & 0,75 & 22 & 0,207 \\
A17 & 0,65 & 22 & 0,228 \\
A18 & 0,55 & 22 & 0,215 \\
A19 & 0,55 & 31 & 0,205 \\
A20 & 0,75 & 31 & 0,175 \\
\hline
\end{tabular}

